The Journal of

Thoracic and Cardiovascular

Surgery

Vol 129, No. 2, February 2005

\title{
Imaging, models, and reality: A basis for anatomic- physiologic planning
}

Gerald D. Buckberg, MD

See related article on page 382 .
From the Department of Cardiothoracic Surgery, UCLA Medical Center, Los Angeles, Calif.

Received for publication June 23, 2004; revisions received Sept 23, 2004; accepted for publication Sept 28, 2004.

Address for reprints: Gerald D. Buckberg, MD, Department of Cardiothoracic Surgery, Room 62-258 CHS, Box 951741, UCLA Medical Center, Los Angeles, CA 90095-1741 (E-mail: gbuckberg@mednet. ucla.edu).

J Thorac Cardiovasc Surg 2005;129:243-5

$0022-5223 / \$ 30.00$

Copyright () 2005 by The American Association for Thoracic Surgery

doi:10.1016/j.jtcvs.2004.09.021

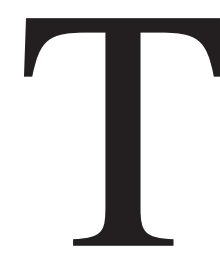

he report in this volume by Walker and associates ${ }^{1}$ is an important attempt to apply diffusion tensor magnetic resonance imaging (MRI) analysis to determine whether the helical fiber orientation of the normal heart geometry is changed when the heart becomes dilated in heart failure and then is restored toward normal as cardiac structure is rebuilt by restoration.

The problem with this study is that the method misses the global disease while focusing on analysis of isolated regions. The flaw in these diffusion tensor MRI studies is failure to use the correct model of the failing heart for the analysis. The basic concept of heart failure is that the dilated heart becomes spherical through a change in the radius of curvature of the ventricular wall. This geometric change characterizes the loaded heart that is stretched rather than the unloaded, decompressed, nonfunctioning heart that is analyzed in this article.

A simple geometric analysis can show that an oblique fiber angle on a surface with a certain radius of curvature will attain a shallower angle as the surface projects itself to one with a larger radius of curvature and with a shorter long axis, as in the case for dilated heart. This change in the angle of fibers is inherent to any such surface deformation. To clarify this important point, we have modeled the ventricle as an ellipsoid of revolution that is fixed to a certain size base (Figure 1). As the heart dilates to a larger ellipsoid (ie, more spherical), one can calculate the decrease in the angle of any helically oriented line on the surface of the ellipsoid. For example, for a $65^{\circ}$ fiber angle, if the short axis of such an ellipsoid changes from 5 to $8 \mathrm{~cm}$, a $10^{\circ}$ change in the fiber angle can be expected. If we allow for $20 \%$ fiber length change, we might see angle changes of up to $20^{\circ}$. Changes as small as $5^{\circ}$ to $10^{\circ}$ can substantially influence the cardiac performance, as has been suggested by Ingels, ${ }^{2}$ Sallin, ${ }^{3}$ and others. In fact, the role of restoration is to reestablish the normal geometry (smaller radius of curvature) and thus normalize the fiber angle by rebuilding the conical chamber.

MRI methods are carefully applied in dead shrunken hearts subjected to extensive regional analysis, but the reality is that imaging studies must relate to how these hearts relate to the live dilated ventricular chamber in cardiac failure. Reporting of 200,000 helix angle measurements in hearts with the wrong geometry does not compensate for conceptual problems that stem from model limitations. The essential criteria of reproducing the less radial oblique angles of curvature seen in congestive heart failure is critical and must now be done subsequently. Sometimes our fascination with the output of new imaging methods overshadows the basic need for using these methods in appropriate models to aid direct transition to clinical events.

The definition of congestive heart failure is related to how the loaded spherical chamber alters the left ventricular end-systolic and end-diastolic volume relationships. These values must be reported for the normal, failing, and restored heart, and 

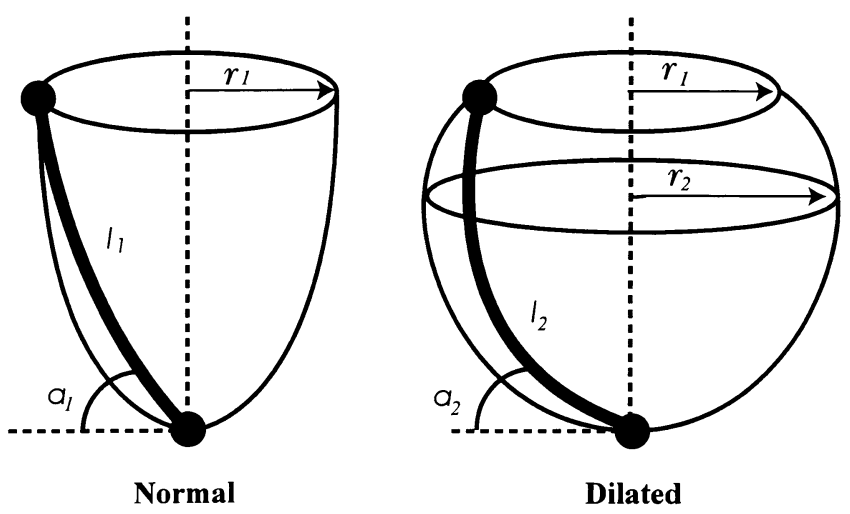

Figure 1. Comparison of ellipsoid and less ellipsoid (more spherical) ventricle. The base, or $r 1$, is unchanged and reflects the mitral filling site. The midchamber ( $r 2$ ) widens, the length (I) and radius ( $r$ ) increase, and the fiber angle degree (a) changes.

such geometric changes must be rebuilt into future analysis of the dead hearts that were studied. Clearly, the authors recognized these limits, for they are summarized under "Limitations." The validity of any study for a clinical heart failure model must use a chamber that is stretched. This model of correct geometry can be accomplished by using an intracavitary balloon in dead hearts and by investigators using volumes that simulate the living values they measured in normal, failing, and restored chambers. Instead, as shown in Figure 2, all studies were done on an unloaded conical chamber that was shrunken by means of decompression during the MRI analysis.

Analysis of the chamber tracings in Figure 3 in the author's text (Figure 2 in this report) shows the anatomy of the dead heart and defines a remarkable similarity to what might be expected in the viable unloaded chamber by means of rebuilding. Consequently, the capacity of rebuilding normal geometry to restore the helix is supported by this study because a conical formation exists in all of the decompressed hearts studied. Proper interpretation of results must be linked to studying a reproduction of geometry of cardiac failure and how this is changed by surgical intervention.

Claude Barnard, in his volume entitled An Introduction to the Study of Experimental Medicine, ${ }^{4}$ contrasted the anatomist, who looks at the nonliving structure and deduces function, with the physiologist, who looks at life and adds other sciences, with anatomy as only one part. This imaging report (simulating anatomy) reconstructs an epicardial angle in the dead heart, and these selection criteria must be replaced by making the epicardium reflect the measured (physiologic) sizes in either systole or diastole in each phase of normality, failure, and repair.

The recent studies by Suma and colleagues ${ }^{5,6}$ define a clinical similarity to this limitation of MRI application to the wrong model of cardiac failure. Instead of MRI, echo- cardiographic studies of wall motion were made in (1) the distended ventricle and (2) after the heart was decompressed by means of volume reduction, and structural and functional changes were contrasted. ${ }^{6}$ Distention caused a loss of regional wall motion, and decompression by means of partial bypass allowed contractile motion to become visible in viable segments. These structural and functional findings allowed proper site selection for volume reduction and led to subsequent clinical improvement. In these patients global cardiac geometry is changed in the distended heart, and the fiber angles in the main pathways should follow this architectural change. Conversely, the dead shrunken heart was studied by means of tensor diffusion in this experimental report, and thereby incorrect geometry of decompression, rather than the correct architectural stretch of cardiac failure, is introduced.

The diffusion tensor imaging studies define the reproducibility of the helix in both the global structure and in the subelements on the basis of this MRI analysis. Aside from congestive heart failure, surgeons also see acute examples of how global architecture is stretched by means of dilation, a configuration in which fiber orientation of the predominant pathways can be made more spherical and function becomes impaired. Such an operating room event occurs during a protamine reaction, in which the chamber dilates and function is immediately impaired. Simply restoring normal architecture by instituting decompression through restarting bypass will usually immediately restore contraction. A chronic example was also shown by Levin and associates, ${ }^{7}$ who defined how the abnormal pressure-volume relationships of the dilated heart can be returned to normal by means of left ventricular decompression with a left ventricular assist device for 3 months. To understand proper fiber orientation, we must study both the failing global sphere and the decompressed cone because reproduction of both architectural models is needed to match clinical events.

The authors reproduce and define the fiber angle helix of approximately $1-\mathrm{mm}$ segments in the normal ventricle. The clinical capacity of changing volume to restore function is clear in the septum, which does not function when distended, but contractile function is immediately restored after decompression by means of ventricular rebuilding in ischemic dilated cardiomyopathy. ${ }^{8}$ During clinical reconstruction, both shape and volume are important considerations. The shrunken rebuilt chamber shown in the author's Figure 3, entitled "Plication," has normal architecture but postoperatively might have problems related to volume restriction, despite its conical shape. Occasionally, patients might experience low cardiac output that is not related to loss of helical fiber angle but rather to excessive cavity decompression. Consequently, the normal helix is returned, but residual intracavitary volume might be wrong.

The authors did not follow the central theme of the cited literature $^{9-11}$ on the architectural change from the normal 
A

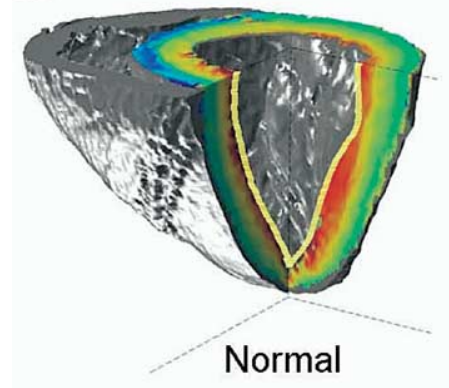

B

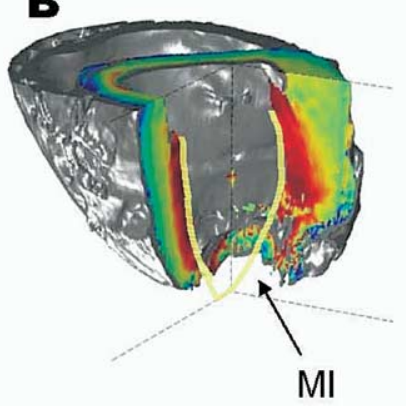

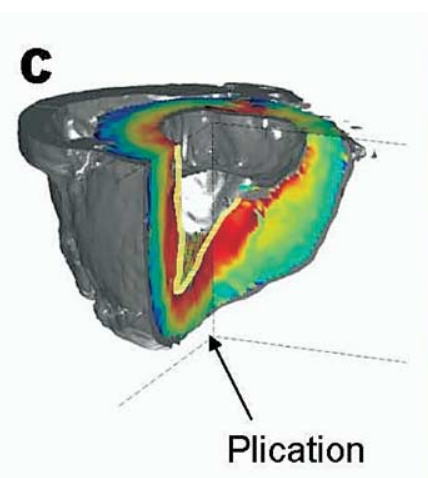

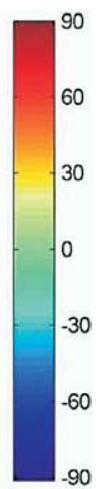

"all conical"

Figure 2. The conical appearance of each decompressed ventricle undergoing MRI diffusion tensor imaging analysis. Note the normal myocardial infarction (MI) and restored (Plication) chambers. Each left ventricular chamber and wall shape has a similar cone configuration.

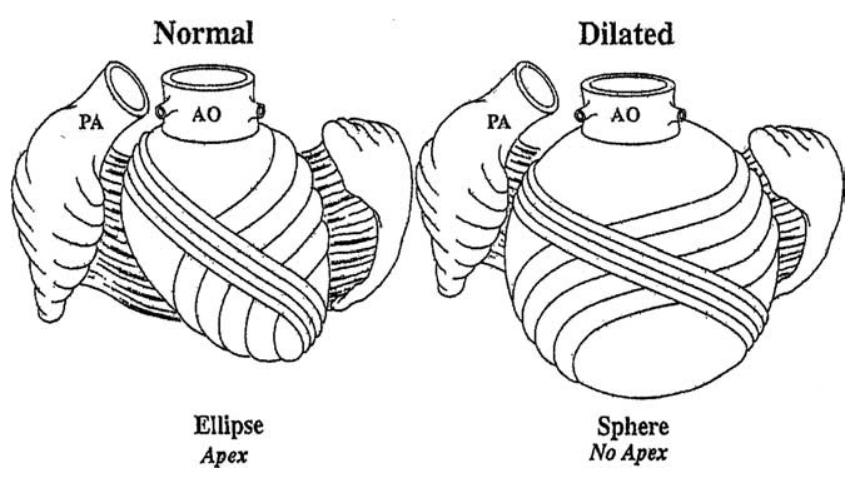

Figure 3. Ventricular shape in the normal elliptical heart (left) and the dilated spherical heart (right). This image shows the different appearance of the underlying oblique apical loop that becomes sphere shaped with dilation. Major fiber orientation begins to resemble the transverse basal loop and the outer cardiac wrap. The size and shape changes shown in Figure 1 resemble these apical loop configurations.

ellipse toward stretch during heart failure, whereby the global fiber orientation of the conical helix of the normal heart develops a spherical chamber configuration, as shown in Figure 3. Inferred is that the myofiber helix of fibers within this global architecture might be normal, but the major fiber pathways carrying them can be changed by dilation. The suggestion is that expected regional function can be regained if the spherical geometry is restored to a conical configuration. Hopefully, this concept can be tested if this MRI study is redone with architectural models that are normal and then altered to reflect the sphere-shaped dimensions of the failing heart and that finally are restored surgically to the normal shape. Avoidance of studying only the decompressed shrunken ventricle must be done to enhance our learning if changes in conical anatomy alter the myofiber helix of the stretched heart and if restoration is successful in rebuilding myofiber helical normality.

\section{References}

1. Walker JC, Guccione JM, Jiang Y, Zhang P, Wallace AW, Hsu EW, et al. Helical myofiber orientation after myocardial infarction and left ventricular surgical restoration in sheep. J Thorac Cardiovasc Surg. 2005:129:382-90.

2. Ingels NB Jr. Myocardial fiber architecture and left ventricular function. Technol Health Care. 1997;5:45-52.

3. Sallin EA. Fiber orientation and ejection fraction in the human ventricle. Biophys J. 1969;9:954-64.

4. Bernard C. An introduction to the study of experimental medicine. New York: Dover Publications; 1957.

5. Suma H, Isomura T, Horii T, Sato T, Kikuchi N, Iwahashi K, et al. Nontransplant cardiac surgery for end-stage cardiomyopathy. J Thorac Cardiovasc Surg. 2000;199:1233-45.

6. Suma H, RESTORE Group. Left ventriculoplasty for nonischemic dilated cardiomyopathy. Semin Thorac Cardiovasc Surg. 2001;13:514-21.

7. Levin HR, Oz MC, Chen JM, Packer M, Rose EA, Burkhoff D. Reversal of chronic ventricular dilation in patients with end-stage cardiomyopathy by prolonged mechanical unloading. Circulation. 1995;91:2717-20.

8. Athanasuleas CL, Stanley AWH Jr, Buckberg GD. Restoration of contractile function in the enlarged left ventricle by exclusion of remodeled akinetic anterior segment: surgical strategy, myocardial protection, and angiographic results. J Card Surg. 1998;13:418-28.

9. Buckberg GD. Basic science review: the helix and the heart. $J$ Thorac Cardiovasc Surg. 2002;124:863-83.

10. Buckberg GD, Coghlan HC, Torrent-Guasp F. The structure and function of the helical heart and its buttress wrapping. V. Anatomic and physiologic considerations in the healthy and failing heart. Semin Thorac Cardiovasc Surg. 2001;13:358-85.

11. Buckberg GD, Coghlan HC, Torrent-Guasp F. The structure and function of the helical heart and its buttress wrapping. VI. Geometric concepts of heart failure and use for structural correction. Semin Thorac Cardiovasc Surg. 2001;13:386-401. 